\title{
Structural basis of redox sensing by a cyanobacterial transcription regulator Bin Li ${ }^{1}$ \\ ${ }^{1}$ No affiliation given \\ leonbli@umich.edu
}

Thioredoxins play a crucial role in maintaining the redox homeostasis of photosynthetic organisms. In the heterocystous cyanobacterium Nostoc sp. PCC7120, the expression of thioredoxin TrxA2 is induced by $\mathrm{H} 2 \mathrm{O} 2$ and regulated by an ArsR/SmtB type transcription factor, RexT. Different from other members of the ArsR/SmtB family which typically bind metal ions, RexT uses an intramolecular disulfide bond to reversibly bind and release DNA. Despite the importance of thioredoxin in oxidative stress response, the molecular details of its regulation by RexT using a disulfide bond are not well understood. Here, we present two high-resolution crystal structures of RexT in its reduced and $\mathrm{H} 2 \mathrm{O} 2$-treated states to $1.95 \AA$ and $2.09 \AA$ resolution, respectively. These structures reveal that RexT showcases a winged helix-turn-helix fold typical of the ArsR/SmtB family, functions as a homodimer, and forms a disulfide bond between Cys 40 and Cys41 in the oxidized state. The importance of these residues was corroborated using site-directed mutagenesis and a $\mathrm{H} 2 \mathrm{O} 2$ consumption assay. Furthermore, a substrate channel for $\mathrm{H} 2 \mathrm{O} 2$ was identified and key residues implicated in $\mathrm{H} 2 \mathrm{O} 2$ binding and activation were verified. Finally, bioinformatics analysis of the ArsR/SmtB family indicate that the vicinal disulfide "redox switch" may be a unique feature for certain cyanobacteria, especially the Nostocales order, presenting an interesting case of how a metal binding scaffold can be repurposed for redox sensing. 